\title{
GÉNEROS DE HONGOS NECROFÍTICOS ASOCIADOS A HOJAS DE Spartina argentinensis Parodi Y Panicum prionitis Ness en Argentina
}

\section{NECROPHITIC FUNGAL GENERA ASSOCIATED TO LEAVES OF Spartina argentinensis Parodi AND Panicum prionitis Nees in Argentina}

\author{
Emiliano Jozami ${ }^{{ }^{*}}$, Rosanna N. Pioli1 ${ }^{1,2}$, y Susana R. Feldman ${ }^{1,2}$ \\ ${ }^{1}$ Biodiversidad Vegetal y Microbiana, Facultad de Ciencias Agrarias, Universidad Nacional Rosario, \\ Parque Villarino, Zavalla, Argentina. \\ ${ }^{2}$ Consejo de Investigaciones de la Universidad Nacional Rosario. IICAR, Biodiversidad Vegetal y Micro- \\ biana, Facultad de Ciencias Agrarias, Universidad Nacional Rosario, Parque Villarino, Zavalla, Argen- \\ tina. rosanna.pioli@gmail.com, sfeldman@unr.edu.ar \\ *Autor para correspondencia E-mail: ejozami@unr.edu.ar
}

\section{RESUMEN}

Los pastizales de Spartina argentinensis y de Panicum prionitis son comunidades herbáceas utilizadas para la producción ganadera en Argentina. En estas comunidades se acumula gran cantidad de biomasa senescente no aprovechable por el ganado por su baja digestibilidad, siendo la quema una práctica recurrentemente utilizada para estimular el rebrote de pasto más tierno. Este tipo de biomasa podría usarse para producir bioetanol, pero para ello es necesario hacer un pretratamiento para aumentar la eficiencia del proceso de hidrólisis. Un posible pretratamiento es el uso de enzimas ligninolíticas que son comúnmente producidas por algunos grupos de hongos. En este trabajo, se relevaron los géneros fúngicos asociados a ambas especies botánicas presentes en distintas localidades y con diferente grado de descomposición. Se encontró predominancia de géneros fúngicos con citada capacidad ligninolítica tales como Alternaria, Aspergillus, Chaetomium, Cladosporium. Epicoccum, Fusarium, Nigrospora, Penicillium, Pestalotia y Phoma. La mayoría de las mismas se encontraron en ambos hospedantes. A partir de estos hongos podrían obtenerse enzimas ligninolíticas que contribuirían a mejorar la eficiencia de la producción de bioetanol lignocelulósico.

Palabras clave: bioetanol, biomasa lignocelulósica, lignina.

\section{ABSTRACT}

Spartina argentinensis and Panicum prionitis are herbaceous grasses that dominate many communities used for livestock production systems. High amounts of biomass accumulate in these communities without being grazed by cattle due to their low digestibility. Hence, farmers often burn these rangelands in order to stimulate higher quality regrowth. This type of biomass could be used to produce bioethanol. As a biomass pretreatment is necessary to increase the efficiency of the hydrolysis process. The use of ligninolytic enzymes commonly produced by some fungi could be an alternative. In this paper, the fungal genera present in both grasses from different locations and with different degrees of decomposition were surveyed. According to the literature, the following isolated fungal genera show ligninolytic capacity: Alternaria, Aspergillus, Chaetomium, Cladosporium, 
Epicoccum, Fusarium, Nigrospora, Penicillium, Pestalotia y Phoma. Most of them were found in both hosts. Ligninolytic enzymes could be obtained from these fungi in order to improve the efficiency of ethanol production of this kind of biomass.

Key words: bioethanol, lignin, lignocellulosic biomass

\section{INTRODUCCIÓN}

El consumo anual de energía primaria mundial supera los $500 \mathrm{EJ}\left(1 \mathrm{EJ}=1 \times 10^{16} \mathrm{~J}\right)$ y se espera que para el año 2100 ascienda a 1275-1500 EJ (Reilly y Peltsev, 2009). De ese total, el $85 \%$ proviene de fuentes de energía no renovables que en algún momento deberán ser reemplazadas por fuentes renovables. La producción de estas últimas se ha visto incrementada principalmente a partir de materias primas usadas para la alimentación humana o del ganado. Asimismo, debido a que las mismas son escasas, surge la necesidad de encontrar fuentes renovables alternativas que no compitan con la producción de alimentos (Khwaja, 2010; Rathmann et al., 2010).

La celulosa es el compuesto orgánico más abundante en el planeta. Por ello existe gran interés en evaluar el uso de diversas fuentes de biomasa lignocelulósica como fuente de energía. Una alternativa es la producción de bioetanol como resultado de la fermentación de la glucosa obtenida a partir de la hidrólisis de la celulosa. No obstante, la eficiencia de hidrólisis se ve reducida por la presencia de lignina que impregna a la matriz de celulosa y hemicelulosa y disminuye la accesibilidad del sustrato por parte de las enzimas hidrolíticas (Gomez et al., 2008).

Spartina argentinensis Parodi (espartillo) y $\mathrm{Pa}$ nicum prionitis Ness. (paja brava) son gramíneas perennes que ocupan grandes extensiones en la República Argentina, poseen metabolismo fotosintético $\mathrm{C} 4$ y propagación tanto sexual como clonal, por la presencia de rizomas. S. argentinensis es la dominante fisonómica de comunidades denominadas espartillares, que ocupan los Bajos Submeridionales y la cuenca sin desagüe de la laguna Mar Chiquita, totalizando aproximadamente $30.000 \mathrm{~km}^{2}$ (Feldman y Lewis, 2005). Los pajonales de $P$. prionitis se encuentran en planicies de inundación del Río Paraná (Franceschi et al., 1989).

En ambos tipos de comunidades, la actividad ganadera bovina predomina como sistema de producción. Estas especies poseen elevada productividad primaria neta aérea, pero tienen baja digestibilidad (Luisoni et al., 2010). En consecuencia, la producción física de estos sistemas productivos difícilmente logra superar $20 \mathrm{~kg}$ de carne $\mathrm{ha}^{-1} \mathrm{año}^{-1}$. Una práctica común por parte de los productores ganaderos es la quema de estos pastizales con el fin de promover el rebrote de mayor digestibilidad. Las plantas rebrotan rápidamente (Feldman y Lewis, 2007) pero a los pocos meses sus hojas están lignificadas, por lo cual se vuelve a alcanzar la situación previa a la quema. En consecuencia, las quemas son disturbios recurrentes en estos ambientes. El material senescente que se acumula entre quemas está conformado, casi en su totalidad, por hojas en distintos grados de descomposición generada principalmente por la acción fúngica.

La biomasa lignocelulósica de $P$. prionitis y $S$. argentinensis podría aprovecharse como materia prima para la obtención de bioetanol de segunda generación. Para este fin, el alto porcentaje de lignina de ambas especies es la principal barrera a vencer (Himmel et al., 2007; Gómez et al., 2008). Existe información sobre descomposición de especies del género Spartina creciendo en costas marinas, donde se señala la relevancia que tienen los hongos en el proceso (Gessner y Goos, 1973; Newell et al., 2000; Newell y Porter, 2000; Newell 2001a; Newell 2001b; Buchan et al., 2002; Kohlmeyer y Volkmann-Kohlmeyer, 2002; Blum et al., 2004; Lyons et al., 2005; Lyons et al., 2010) puesto que este grupo de organismos se destaca por su capacidad ligninolítica (Benner et al., 1986; Newell and Porter, 2000).

En Argentina, es muy escasa la información existente sobre este tema. En un relevamiento de las especies fúngicas asociadas a hojas senescentes de $S$. argentinensis procedentes de un espartillar de San Cristóbal (Santa Fe, Argentina), se observaron diferencias en la micota asociada y aislada en muestras con diferente nivel de degradación (Jozami et al., 2011).

El objetivo del presente trabajo fue identificar géneros fúngicos que se encuentran asociados a las hojas de $S$. argentinensis y $P$. prionitis en diferentes grados de descomposición y recolectadas en distintas localidades para ser evaluados como futuras fuentes de enzimas ligninolíticas potencialmente utilizables en procesos de producción de bioetanol.

\section{MATERIALES Y MÉTODOS}

\section{Recolección y procesamiento de las muestras}

Las muestras de hojas de $S$. argentinensis se recolectaron en la localidad de Pérez (32 $58^{\prime} \mathrm{S}$; $60^{\circ} 47^{\prime} \mathrm{W}$ ) y margen noroeste del arroyo Ludueña 
( $\left.32^{\circ} 56^{\prime} \mathrm{S} ; 60^{\circ} 48^{\prime} \mathrm{W}\right)$. Las muestras de $P$. prionitis se obtuvieron de un pajonal ubicado en una isla frente a la ciudad de Rosario ( $32^{\circ} 52^{\prime} \mathrm{S} ; 60^{\circ} 35^{\prime} \mathrm{W}$ ).

Para cada especie vegetal y sitio se recolectaron del suelo hojas con dos grados diferentes de descomposición (PD: poco degradadas, hojas cloróticas, coloración amarillenta; MD: muy degradadas, coloración amarronada, estructura quebradiza y en contacto más íntimo con el suelo), que se mantuvieron a $4^{\circ} \mathrm{C}$ hasta su procesamiento. En la Tabla 1 se indica el número de muestras obtenidas para cada combinación de sitio y grado de descomposición.

Se pesó $1 \mathrm{~g}$ de cada muestra cortada en segmentos de $1 \mathrm{~cm}$ y se desinfectó superficialmente durante 30 segundos en una solución de hipoclorito al 0,5\%, en cámara de flujo laminar. Posteriormente se colocó cada muestra en $100 \mathrm{~mL}$ de agua estéril con una gota de tensioactivo no iónico (Tween 20) en Erlenmeyer de $250 \mathrm{~mL}$ que se agitaron durante 30 minutos a $\sim 300 \mathrm{rpm}$ y se obtuvieron diluciones efectivas entre 1 x $10^{-3}$ y 1 x $10^{-5}$ en condiciones de asepsia. Cada muestra procesada se sembró en 3 placas de Petri con medio agar papa glucosado acidulado con el agregado de una gota de ácido láctico al $25 \%$ por placa (APGA). La siembra consistió en distribuir $1 \mathrm{~mL}$ de cada dilución (previo agitado con vortex) en cada placa con APGA (Pioli et al., 1994; Luque et al., 2005; Uboldi et al., 2010). La incubación se realizó en estufa de cultivo, a $26 \pm 1^{\circ} \mathrm{C}$ y oscuridad, durante 7 días.

Los géneros fúngicos presentes se identificaron por observación de la macro y micro-morfología (colonias y estructuras reproductivas, respectivamente) (Alexopoulos et al., 1996; Agrios et al., 2005; Barnett y Hunter, 1998; Leslie et al., 2006).

\section{Determinación de micota total y abundancia}

La abundancia es una medida cuantitativa expresada como número de unidades formadoras de colonias (UFC) para cada género por repetición. Las UFC totales o micota total (MT) se determinaron para cada uno de los sitios de origen de las muestras, hospedante y grado de descomposición de las hojas.

\section{Análisis multivariados a partir de datos de prevalencia}

La prevalencia es una medida cualititativa que refiere a la proporción (o porcentaje) de unidades muestrales colonizadas por los hongos, estimadas en base a la presencia (1) o ausencia (0) de cada género fúngico en cada una de las repeticiones de las muestras obtenidas por localidad de procedencia, hospedante y grados de descomposición. En base a estos datos se construyó una matriz que se analizó mediante un análisis de componentes principales (Peck, 2010) y un procedimiento de permutación de respuestas múltiples (MRPP, del inglés "multiple response permutation procedu$\mathrm{re}^{\prime \prime}$ ) (McCune and Grace, 2002). Los valores de riqueza (S: número de géneros fúngicos presentes por repetición) se compararon mediante ANOVA previa transformación de $\operatorname{los}$ datos $(\log X+0,001)$ (Balzarini et al., 2008).

\section{Análisis bibliográfico}

Se buscó en la bibliografía si los géneros fúngicos aislados a partir de hojas de S. argentinensis o $P$. prionitis estan o no citados como hongos ligninolíticos.

\section{RESULTADOS}

\section{Determinación de abundancia y micota total}

Se registraron 13 géneros fúngicos (Tabla 2). Aspergillus, Penicillium, Alternaria, Cladosporium, Nigrospora y Epicoccum estuvieron presentes en todos los sitios y grados de descomposición. Drechslera y Phyllosticta mostraron un comportamiento similar respecto al hospedante y localidad, sin embargo no fueron aislados a partir de residuos poco degradados de $S$. argentinensis de Ludueña. Por su parte, Fusarium y Phoma sólo se encontraron asociados a $S$. argentinensis, mientras que Pestalotia y un hongo con micelio y picnidios no definido morfológica pero sí identificado molecularmente como Phomopsis sp. (Pioli, 2015) son los que predominaron en $P$. prionitis.

Tabla 1. Número de muestras de hojas recogidas, discriminadas por hospedante, sitio y grado de descomposición.

Table 1. Number of leaf samples, considering hosts, site and degree of decomposition.

\begin{tabular}{llcc}
\hline Hospedante & Sitio & PD & MD \\
\hline S. argentinensis & Pérez & 5 & 5 \\
S. argentinensis & Ludueña & 7 & 9 \\
P. prionitis & Isla & 5 & 5 \\
\hline
\end{tabular}

PD: hojas poco degradadas; MD: hojas muy degradadas. 
Tabla 2. Abundancia de cada género fúngico y micota total asociados a S. argentiniensis y P. prionitis, expresadas en UFC medio por sitio (Pérez, Ludueña. Isla) y grado de descomposición (PD: hoja poco descompuesta; MD: hoja muy descompuesta).

Table 2. Abundance of fungi genera and total mycota associated with $S$. argentiniensis and $P$. prionitis, expressed in UFC (colony formation units) per site (Pérez, Ludueña. Isla) and degree of decomposition (PD: leaf with low decomposition; MD: highly decomposed leaf).

\begin{tabular}{|c|c|c|c|c|c|c|}
\hline \multirow{3}{*}{$\begin{array}{l}\text { Localidad } \\
\text { Género }\end{array}$} & \multicolumn{3}{|c|}{ Spartina argentinensis } & \multicolumn{3}{|c|}{ Panicum prionitis } \\
\hline & \multicolumn{2}{|c|}{------Pérez ------ } & \multicolumn{2}{|c|}{------ Ludueña ------ } & \multicolumn{2}{|c|}{------ Isla ----- } \\
\hline & PD & MD & PD & MD & PD & MD \\
\hline Alternaria & 2,92 & 1,86 & $\begin{array}{l}-- \text { UFC } \\
0,71\end{array}$ & 0,08 & 1,21 & 6,40 \\
\hline Aspergillus & 0,62 & 2,00 & 0,57 & 0,08 & 0,36 & 0,40 \\
\hline Chaetomium & & 0,14 & & & & \\
\hline Cladosporium & 0,92 & 2,64 & 0,10 & 0,38 & 0,29 & 0,47 \\
\hline Drechslera & 0,08 & 0,14 & & 0,08 & 0,93 & 0,87 \\
\hline Ерісоссит & 0,23 & 0,14 & 0,05 & 0,04 & 0,14 & 0,13 \\
\hline Fusarium & 2,46 & 1,00 & 3,14 & 0,13 & & \\
\hline Nigrospora & 0,54 & 0,50 & 0,24 & 1,92 & 1,57 & 0,60 \\
\hline Penicillium & 0,69 & 0,86 & 0,86 & 0,46 & 0,14 & 2,13 \\
\hline Pestalotia & & & & & 0,43 & 2,07 \\
\hline Phoma & 0,62 & 0,57 & 0,05 & & & \\
\hline Phomopsis & & & 0,86 & & 2,21 & 1,47 \\
\hline Phyllosticta & 0,77 & 0,36 & & 0,79 & 1,50 & 7,93 \\
\hline MT & 9,85 & 10,21 & 6,57 & 3,96 & 8,79 & 22,47 \\
\hline
\end{tabular}

\section{Análisis multivariados a partir de datos de prevalencia}

No se observó un patrón definido en la ubicación de las muestras en el diagrama de dispersión en plano de los primeros dos ejes de la variancia total. Las muestras de Spartina sp. (Pérez y Ludueña con distinto grado de descomposición) se ubicaron en los cuatro cuadrantes, mientras que las de Panicum sp. prevalecieron en los cuadrantes 1,2 y 3, con sólo tres muestras en el cuadrante 4. Esta tendencia determinó que los primeros dos ejes del análisis de componentes principales absorbieran sólo el 32\% de la variancia total (Fig. 1).

El análisis MRPP detectó diferencias entre sitios ( $p=0,00000000)$, las que podrían atribuirse a los valores de riqueza $(p<0,0001)$ (Tabla 3$)$. El único sitio donde se encontraron diferencias entre los grados de descomposición fue en la Isla $(P$. prionitis) donde las muestras MD mostraron valores superiores de riqueza. Las muestras de Isla MD, junto a las de Pérez MD y PD, presentaron la mayor riqueza de géneros fúngicos, mientras que el valor más bajo se registró en Ludueña MD, sobre hojas de $S$. argentinensis.

\section{Relevamiento bibliográfico}

Al buscar en la bibliografía, se encontraron 11 trabajos en los cuales se mencionaba la presencia de enzimas ligninolíticas en los géneros fúngicos aislados en el presente estudio (Tabla 4).

\section{DISCUSIÓN}

La lignina es el componente más recalcitrante de los materiales lignocelulósicos y actúa como una barrera dificultando la acción de las enzimas celulolíticas sobre la pared celular, protegiendo de este modo su estructura (Himmel et al., 2007; Gómez et al., 2008; Sánchez, 2009). Considerando que la celulosa es el compuesto orgánico más abundante en la tierra, los hongos necrofíticos ligninolíticos cumplen un rol importante en el ciclado de la materia al aumentar la accesibilidad de los carbohidratos estructurales por parte de las enzimas celulolíticas.

Los hongos de podredumbre suave (HPS) son ascomicetes o sus estados imperfectos y presentan actividad ligninolítica (Tuomela et al., 2000). Esta denominación (suave) se debe a su aparente menor eficiencia en el proceso de degradación de lignina presente en la madera, comparado con los basidiomicetes (Sin et al., 2002). Sin embargo, a pesar de su mayor eficiencia, la relevancia de los basidiomicetes como ligninolíticos en el suelo es cuestionable debido a su mayor sensibilidad a las condiciones de anaerobiosis, humedad, $\mathrm{pH}$ y temperatura reinantes (Tuomela, 2002). Otros autores señalan que la relación $\mathrm{C} / \mathrm{N}$ del suelo también actúa como limitante para el metabolismo de los basidiomicetes con actividad ligninolítica (López et al. 2006). Los HPS degradan madera en 


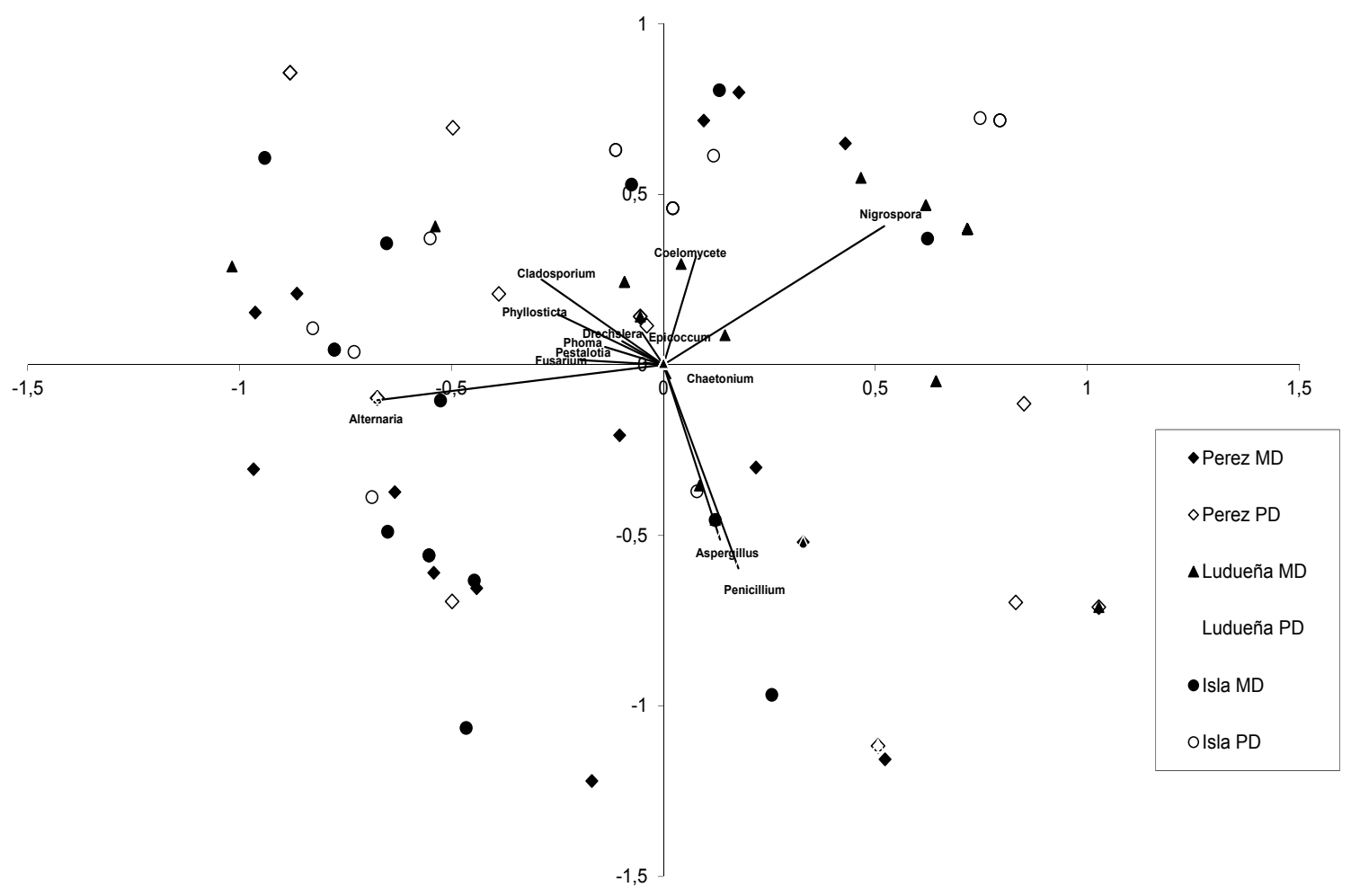

Fig. 1. Diagrama de dispersión en el plano de los ejes 1 y 2 del análisis de componentes principales de la prevalencia de los 13 géneros fúngicos en S. argentinensis (Ludueña y Pérez) y $P$. prionitis (Isla) con diferentes grados de descomposición (PD: poco degradadas; MD: muy degradadas).

Fig. 1. Scatter diagram of axes 1 and 2 of principal components analysis of the 13 fungi genera in leaves of S. argentinensis (Ludueña y Pérez) and P. prionitis (Isla) with different degrees of decomposition (PD: low degraded; MD: highly degraded).

Tabla 3. Riqueza (S) de géneros fúngicos aislados a partir de muestras de hojas de S. argentinensis (Pérez, Ludueña) y $P$. prionitis (Isla) (PD: poco degradadas; MD: muy degradadas. Valores seguidos por igual letra no difieren significativamente (ANOVA, $p<0,05$ ).

Table 3. Richness (S) of fungi genera isolated from leaf samples of S. argentinensis (Pérez, Ludueña) and $P$. prionitis (Isla) (PD: low degraded; MD: highly degraded.

Values followed by the same letter do not differ significantly (ANOVA, $p<0.05$ ).

\begin{tabular}{ll}
\hline Sitio & \multicolumn{1}{c}{ S } \\
\hline Isla MD & $3,47 \mathrm{a}$ \\
Pérez MD & $3,07 \mathrm{a}$ \\
Pérez PD & $2,92 \mathrm{ab}$ \\
Isla PD & $2,64 \mathrm{bc}$ \\
Ludueña PD & $2,00 \mathrm{bc}$ \\
Ludueña MD & $1,67 \mathrm{c}$ \\
\hline
\end{tabular}


Tabla 4. Referencias bibliográficas donde se mencionan la presencia de enzimas ligninolíticas ${ }^{1}$ en géneros de hongos aislados a partir de hojas de S. argentinensis y P. prionitis.

Table 4. References that describe the presence of ligninolitic enzymes in fungi genera isolated from S. argentinensis and $P$. prionitis leaves.

\begin{tabular}{lll}
\hline Especie & Enzima & Referencia \\
\hline Alternaria alternata & Lac & Kellner et al., 2009 \\
Aspergillus niger & Lac & Ilyas y Rehman, 2013 \\
Aspergillus niger & Fe & Sánchez, 2009 \\
Chaetomium thermophilium & Lac & Amitai et al., 1998 \\
Cladosporium cladosporioides & MnP, LiP, Lac & Bonugli-Santos et al., 2010 \\
Epicoccum purpurascens & Lac & Atalla et al., 2010 \\
Fusarium merismoides & Ae & Sánchez, 2009 \\
Fusarium oxysporum & Lac & Falcón et al., 1995 \\
Fusarium oxysporum & AAD, LiP & Mönkemann et al., 1997 \\
Fusarium proliferatum & Lac, AAO & Regalado et al., 1999 \\
Fusarium solani & Lac & Falcón et al., 1995 \\
Fusarium solani & Lac, MnP, AAO, MiP & Saparrat et al., 2000 \\
Nigrospora sp. & Lac & Ilyas y Rehman, 2013 \\
Penicillium chrysogenum & Lac & Falcón et al., 1995 \\
Penicillium simplicissimum & Lac & Zeng et al., 2006 \\
Pestalotia bicolor & LiP & Patel et al., 2007 \\
Pestalotia oxyanthi & Lac & Falcón et al., 1995 \\
Phoma sp. & Lac & Junghanns et al., 2009 \\
\hline
\end{tabular}

${ }^{1}$ AAO: aril alcohol oxidasa; Lac: Lacasa; LiP: lignina peroxidasa; MnP: manganeso peroxidasa; MiP: peroxidasa manganeso independiente; AAD: arilalcohol dehidrogenasa; Ae: acetil esterasa; Fe: feruloil esterasa.

ambientes severos para los basidiomicetes. También se los ha reportado como descomponedores de hojarasca y lignina marcada con C14 de tallos de maíz y de rastrojo de trigo (Tuomela, 2000). En concordancia con esto, el presente estudio muestra que los 13 géneros fúngicos identificados sobre $S$. argentinensis y $P$. prionitis, corresponden a la División Ascomycota, cuyos miembros tienen un ciclo biológico con una fase sexual (teleomorfa) y asexual (anamorfa). Por ello, algunos aislamientos fueron descritos e identificados en su fase teleomorfa o peritecial, tal el caso de Chaetomium; y/mientras los aislamientos de los 12 géneros restantes fueron descritos e identificados en su fase anamorfa (Alexopoulos 1996; Agrios, 2005; Llamas Frade y Terrón Alonso, 2005). Todos ellos han sido citados como HPS en trabajos previos.

Los géneros fúngicos aquí reportados, Alternaria, Fusarium (F. semitectum, F. oxysporum), Drechslera (D. dematioidea, D. spp.), Nigrospora y Epicoccum, habían sido aislados sobre propágulos de origen sexual de $S$. argentinensis presentes en la Reserva Federico Wildermuth (31 $57^{\prime} \mathrm{S}$; $61^{\circ} 23^{\prime} \mathrm{O}$ ) (Feldman et al., 2004). Asimismo Aspergillus, Penicillium y $F$. oxysporum fueron registrados sobre hojas de S. argentinensis en descomposición en la localidad de San Cristóbal ( $30^{\circ} 19^{\prime} 41^{\prime \prime}$ S; 61 ${ }^{\circ} 13^{\prime} 37^{\prime \prime}$ O) (Jozami et al., 2011); siendo ambas localidades de la provincia de Santa Fe. Sin embargo, el número de microorganismos fúngicos totales presentes en residuos foliares de San Cristóbal y en ese ciclo de crecimiento, 2011, fue menor, posiblemente debido a la sequía registrada en ese año (Servicio Meteorológico Nacional, 2011). De acuerdo con Blum et al. (2004), estos resultados reflejan el perfil de distribución de las comunidades fúngicas respecto del tipo de especie vegetal, calidad (nivel de degradado) y localidad (ambiente), y permitirían inferir el rol ecológico durante el proceso de degradación de hojas de $S$. argentinensis y $P$. prionitis de dichas poblaciones fúngicas. Por su parte, los resultados obtenidos a partir de hojas de $P$. prionitis en diferentes grado de descomposición fueron coherentes con Kleczewski et al. (2012), quienes informaron que los géneros Alternaria, Epicoccum, Phoma, Phaeosphaeria, Stagonospora y otros ascomicetes fueron los más frecuentemente aislados de esta especie.

Algunos de los géneros hallados en las muestras analizadas, entre ellos Penicillium, Aspergillus, Fusarium, Alternaria y Dreschlera, presentaron además actividad celulolítica en estudios específicos realizados por Luque et al. (2005). En todas 
las muestras, además se encontraron géneros fúngicos previamente citados como ligninolíticos en coincidencia con otros autores. Entre ellos, Boland y Grund (1979), Gessner (1977) y Gessner y Kohlmeyer (1976) revelaron que más de 100 especies de hongos filamentosos son encontrados comúnmente sobre hojas del genero Spartina en descomposición en la costa este de Estados Unidos. Newell et al. (1989) hallaron que la descomposición de S. alternifora se debía principalmente a la acción de hongos ascomicetes. Phaeosphaeria spartinicola y Mycosphaerella sp. serían algunas de las especies más frecuentemente involucradas en el proceso de degradación de hojas del género Spartina (Lyons et al., 2010).

Todas las muestras bajo estudio estuvieron asociadas al menos a un género ligninolítico. En términos generales, se trató de hongos no específicos, es decir presentes en ambos hospedantes lo cual, considerando la alta heterogeneidad de la molécula de lignina (Himmel et al., 2007), es una ventaja teniendo en cuenta su potencial uso para la obtención de bioetanol. Por lo tanto, las especies fúngicas aisladas en $S$. argentinensis y $P$. prionitis en este trabajo, deberían ser consideradas a la hora de estudiar posibles pretratamientos que remuevan la lignina y permitan aumentar la eficiencia de hidrólisis de celulosa de estas fuentes de biomasa.

\section{CONCLUSIONES}

Los hongos responsables de la degradación foliar de $S$. argentinensis y $P$. prionitis corresponden a géneros polífiticos. Se aislaron 13 géneros fúngicos, la mayoría de ellos con actividad ligninolítica ya reportada y cuyas enzimas podrían utilizarse para el pretratamiento de la biomasa. Seis géneros estuvieron presentes en todos los sitios, en ambas especies y para los dos grados de descomposición, poniendo en evidencia su baja especificidad en relación a hospedantes, ambientes y estado del sustrato. Esta característica sería altamente deseable al evaluar posibles pretratamientos biológicos para distintos recursos biomásicos.

\section{LITERATURA CITADA}

Agrios, G. 2005. Plant Pathology. 5th. ed. Department of Plant Pathology, University of Florida. Elsevier Academic Press, London, UK.

Alexopoulos, C.J., and C.W. Mims. 1996. Introductory Micology. 4th. ed. Wiley \& Sons, Inc., New York, USA.
Amitai, G., R. Adani, G. Sod-Moriah, I. Rabinovitz, A. Vincze, H. Leader, and Y. Hadar. 1998. Oxidative biodegradation of phosphorothiolates by fungal laccase. FEBS Letters 438(3):195-200.

Atalla, M.M., H.K. Zeinab, R.H. Eman, A.Y. Amani, and A.A.E.A. Abeer. 2010. Screening of some marine-derived fungal isolates for lignin degrading enzymes (LDEs) production. Agr. Biol. J. N. Am. 1(4):591-599.

Balzarini, M.G., L. Gonzalez, M. Tablada, F. Casanoves, J.A. Di Rienzo, y C.W. Robledo. 2008. Infostat. Manual del Usuario. Editorial Brujas, Córdoba, Argentina.

Barnett, H.L., and B.B. Hunter. 1998. Illustrated Genera of Fungi Imperfecti. 4th. ed. APS Press, USA.

Benner, R., M.A. Moran, and R.E. Hodson. 1986. Biogeochemical cycling of lignocellulosic carbon in marine and freshwater ecosystems: relative contributions of prokaryotes and eukaryotes. Limnol Oceanogr. 31(1):89-100.

Blum, L.K., M.S. Roberts, J.L. Garland, and A.L. Mills. 2004. Distribution of microbial communities associated with the dominant high marsh plants and sediments of the United States East Coast. Microbial Ecol. 48(3):375388.

Boland, G.J., and D.W. Grund. 1979. Fungi from the salt marshes of Minas Basin, Nova Scotia. Proceedings of the Nova Scotian Institute of Science 29:393-304.

Bonugli-Santos, R.C., L.R. Durrant, M. Da Silva, and L.D. Sette. 2010. Production of laccase, manganese peroxidase and lignin peroxidase by Brazilian marine-derived fungi. Enzyme Microb Tech. 46(1):32-37

Buchan, A, S.Y. Newell, J.I.L. Moreta, and M.A. Moran. 2002. Analysis of internal transcribed spacer (ITS) regions of rRNA genes in fungal communities in a southeastern US salt marsh. Microb Ecol. 43(3):329-340.

England, C.A., and E.L. Rice. 1957. A comparison of the soil fungi of a tall-grass prairie and of an abandoned field in central Oklahoma. Bot Gaz. 118(3):186-190.

Falcón, M.A., A. Rodríguez, A. Carnicero, V. Regalado, F. Perestelo, O. Milstein, and G. De La Fuente. 1995. Isolation of microorganisms with lignin transformation potential from soil of Tenerife Island. Soil Biol. Biochem. 27(2):121-126.

Feldman, S.R., and J.P. Lewis. 2007. Effect of fire on Spartina argentinensis Parodi demographic characteristics. Wetlands 27(4):785-793. 
Feldman, S.R., and J.P. Lewis. 2005. Effect of fire on the structure and diversity of a Spartina argentinensis tall grassland. Applied Vegetation Science 8:77-84.

Feldman, S.R., R.N. Pioli, y J.P. Lewis. 2004. Caracterización de los propágulos de origen sexual de Spartina argentinensis Parodi. Revista de Investigaciones de la Facultad de Ciencias Agrarias-UNR 6:65-68.

Franceschi, E.A., D.E. Prado, and J.P. Lewis. 1989. Distribution of herbaceous communities of the river Parana along an elevation gradient after a catastrophic flood. Coenoses 4(1):4753.

Gessner, R.V., and R.D. Goos. 1973. Fungi from decomposing Spartina alterniflora. Can. J. Bot. 51(1):51-55.

Gessner, R.V., and J. Kohlmeyer. 1976. Geographical distribution and taxonomy of fungi from salt marsh Spartina. Can. J. Bot. 54(17):20232037.

Gessner, R.V. 1977. Seasonal occurrence and distribution of fungi associated with Spartina alterniflora from a Rhode Island estuary. Mycologia 69(3):477-491.

Gomez, L.D., C.G. Steele-King, and S.J. Mcqueen-Mason. 2008. Sustainable liquid biofuels from biomass: the writing's on the walls. New Phytol. 178(3):473-485.

Himmel, M.E., S.Y. Ding, D.K. Johnson, W.S. Adney, M.R. Nimlos, J.W. Brady, and T.D. Foust. 2007. Biomass recalcitrance: engineering plants and enzymes for biofuels production. Science 315(5813):802-807.

Ilyas S., and A. Rehman. 2013. Decolorization and detoxification of Synozol red HF-6BN azo dye, by Aspergillus niger and Nigrospora sp. Iranian J. Environ. Health Sci. and Eng. 10(1):12

Jozami, E., R.N. Pioli, R. Depetris, S.R. Feldman, y H. Permingeat. 2011. Relevamiento de hongos asociados al residuo de Spartina argentinensis en San Cristobal, Santa Fe. XIII Congreso y XXXI Reunión Anual Sociedad de Biología de Rosario. 1 y 2 de diciembre de 2011. Sociedad de Biología, Rosario, Argentina.

Junghanns, C., M.J. Pecyna, D. Böhm, N. Jehmlich, C. Martin, M. Von Bergen, and D. Schlosser. 2009. Biochemical and molecular genetic characterisation of a novel laccase produced by the aquatic ascomycete Phoma sp. UHH 5-1-03. Appl. Microbiol. Biot. 84(6):1095-1105.

Kellner, H., P. Luis, B. Schlitt, and F. Buscot. 2009. Temporal changes in diversity and expression patterns of fungal laccase genes within the organic horizon of a brown forest soil. Soil Biol. Biochem. 41:1380-1389.
Khwaja, Y. 2010. Bioenergía y seguridad alimentaria BEFS. El análisis de BEFS para el Peru. El Proyeto BEFS: Apoyo a la política bioenergética en Perú. Medio Ambiente y Recursos Naturales. Documento de Trabajo. FAO, Roma, Italia.

Kleczewski, N.M., J.T. Bauer, J.D. Bever, K. Clay, and H.L. Reynolds. 2012. A survey of endophytic fungi of switchgrass (Panicum virgatum) in the Midwest, and their putative roles in plant growth. Fungal Ecol. 5(7):521-529.

Kohlmeyer, J., and B. Volkmann-Kohlmeyer. 2002. Fungi on Juncus and Spartina: new marine species of Anthostomella, with a list of marine fungi known on Spartina. Mycol. Res. 106(3):365-374.

Llamas Frade, B. y A. Terrón. 2005. Guía de campo de los hongos de la Península Ibérica. Celarayn Edit., León, España.

Leslie, J.L., and B.A. Summerell. 2006. The Fusarium Laboratory Manual. Blackwell Publishing, Ames, Iowa, USA.

López, M.J., M. del Carmen Vargas-García, F. Suárez-Estrella, and J. Moreno. 2006. Biodelignification and humification of horticultural plant residues by fungi. Int. Biodeter. Biodegr. 57(1):24-30.

Lyons, J.I., M. Alber, and J.T. Hollibaugh. 2010. Ascomycete fungal communities associated with early decaying leaves of Spartina spp. from central California estuaries. Oecologia 162(2):435-442.

Lyons, J.I., S.Y. Newell, R.P. Brown, M.A. Moran, and J.P. Lewis. 2005. Screening for bacterialfungal associations in a south-eastern US salt marshusing pre-established fungal monocultures. FEMS Microbiol. Ecol. 54(2):179-187.

McCune, B., and J.B. Grace. 2002. Analysis of ecological communities. Version 5.0. MjM Software Design, Gleneden Beach, Oregon, USA.

Mobberly, D.G. 1956. Taxonomy and distribution of the genus Spartina. Iowa St. College J. of Sci. 30(4):471-574.

Mönkemann, H., U. Hölker, and M. Höfer. 1997. Components of the ligninolytic system of $\mathrm{Fu}$ sarium oxysporum and Trichoderma atroviride. Fuel Process. Technol. 52(1):73-77.

Newell, S.Y., L.K. Blum, C.E. Crawford, T. Dai, and M. Dionne. 2000. Autumnal biomass and productivity of saltmarsh fungi from 29 to 43 degrees north latitude along the United States Atlantic coast. Appl. Environ. Microbiol. 66(1):180-185

Newell S.Y., R.D. Fallon, and J.D. Miller. 1989. Decomposition and microbial dynamics for standing, naturally positioned leaves of the salt-marsh grass Spartina alterniflora. Mar. Biol. 101(4):471-481. 
Newell, S.Y., and D. Porter. 2000. Microbial secondary production from salt-marsh grass shoots, and its known and potential fates. In M.P. Weinstein and D.A. Kreeger (eds.). Concepts and controversies in tidal marsh ecology. Kluwer, Dordrecht, Netherlands.

Newell, S.Y. 2001a. Multiyear patterns of fungal biomass dynamics and productivity within naturally decaying smooth cordgrass shoots. Limnol Oceanogr. 46(3):573-583.

Newell, S.Y. 2001b. Spore-expulsion rates and extents of blade occupation by ascomycetes of the smooth-cordgrass standing decay system. Bot. Marin. 44(3):277-285.

Patel, V.K., R.S.S. Yadav, and K.D.S. Yadav. 2007. Enzymatic characteristics of lignin peroxidases of indigenous lignolytic fungal strains. Part I. Indian J. Biotechnol. 6(4):553.

Pioli, R.N., S. Montico, B. Bonel, S. Toresani, E. Gómez, y V. Bisaro. 1994. Estudio de la relación entre sobrevivencia de patógenos, degradación del rastrojo de trigo y la actividad celulolítica. III Congreso Nacional de Trigo y $1^{\circ}$ Simposio Nacional de Cereales. Junio 1994. p. 225-226. Departamento de Agronomía, Universidad Nacional del Sur, Asociación de Ing. Agr. del Norte de la Provincia de Bs. As., Bahía Blanca, Buenos Aires, Argentina.

Pioli, R. N. 2015. Estudios sobre algunos mecanismos de variabilidad genética fúngica en el complejo Diaporthe - Phomopsis asociado a cultivos de Argentina. Trabajo Final Programa Posdoctoral. UNR, Facultad de Ciencias Agrarias, Rosario, Argentina.

Rathmann, R., A. Szklo, and R. Schaeffer. 2010. Land use competition for production of food and liquid biofuels: An analysis of the arguments in the current debate. Renew Energ. 35(1):14-22.

Regalado, V., F. Perestelo, A. Rodríguez, A. Carnicero, F.J. Sosa, G. De La Fuente, and M.A. Falcon. 1999. Activated oxygen species and two extracellular enzymes: laccase and aryl-alcohol oxidase, novel for the lignin-degrading fungus Fusarium proliferatum. Appl. Microbiol. Biotechnol. 51(3):388-390.
Reilly, J., and S. Peltsev. 2009. Biomass energy and competition for land. In Hertel T., Rose S., Tol R. (eds.). Economic analysis of land use in global climate change policy. Routledge, London, UK.

Sánchez, C. 2009. Lignocellulosic residues: Biodegradation and bioconversion by fungi. Biotechnol. Adv. 27(2):185-194.

Saparrat, M.C.N., M.J. Martínez, H.A. Tournier, M.N. Cabello, and A.M. Arambarri. 2000. Production of ligninolytic enzymes by $\mathrm{Fu}$ sarium solani strains isolated from different substrata. World J. Microbiol. Biotechnol. 16(8-9):799-803.

Servicio Meteorológico Nacional. 2011. Boletín climatológico 2011: XXIII. Disponible en http://www.smn.gov.ar/serviciosclimaticos/ clima/archivo/clim-anual11.pdf. (Consulta 30 diciembre 2015)

Sin, M.K.W., K.D. Hyde, and S.B. 2002. Comparative enzyme production by fungi from diverse lignocellulosic substrates. J. Microbiol. 40(3):241-244.

Tuomela, M. 2002. Degradation of lignin and other 14C-labelled compounds in compost and soil with an emphasis on white-rot fungi. Ph.D. Thesis. Department of Applied Chemistry and Microbiology, University of Helsinki, Helsinki, Finland.

Tuomela, M., M. Vikman, A. Hatakka, and M. Itävaara. 2000. Biodegradation of lignin in a compost environment: a review. Bioresource Technology 72(2):169-183.

Uboldi L., R.N. Pioli, A. Luque, y V. Bisaro. 2010. Efecto del ambiente y las prácticas de manejo de suelo y cultivo sobre la degradación del rastrojo de trigo y las poblaciones fúngicas asociadas. Divulgación de la Producción Científica y Tecnológica. Universidad Nac. Rosario. SCyT. UNR IV:554-557.

Zeng G.M., Y.Y. Yu, H.L. Huang, D.L. Huang, Y.N. Chen, G.H. Huang, and J.B. Li.. 2006. Laccase activities of a soil fungus Penicillium simplicissimum in relation to lignin degradation. World J. Microbiol. Biotechnol. 22(4):317-324. 